\title{
Factores de las percepciones de auditores financieros sobre auditoría del valor razonable
}

\section{Factors of financial auditor's perceptions about fair value auditing}

\author{
Kiara Chau Miyakawa ${ }^{1}$, Julio Hernández Pajares ${ }^{2}$
}

\begin{abstract}
RESUMEN
La incorporación del modelo de medición de valor razonable (VR) en las NIIF han generado grandes esfuerzos y desafíos para los auditores financieros en el proceso de auditoría de las estimaciones del VR en la preparación de los estados financieros. El objetivo de la investigación es conocer la percepción de los auditores financieros sobre el proceso de auditoría del VR considerando los aspectos más relevantes de las normas NIIF 13 y NIA 540. Otros objetivos son percibir la opinión sobre los retos que implican estas normas y la contribución en la imagen fiel de la información financiera, comprensión de las estrategias que mejoren el proceso de auditoría del VR y analizar la relación de estas percepciones con los factores de nivel académico, experiencia, género y sector especializado de los auditores. Para cumplir dichos objetivos se realizó un estudio exploratorio y cuantitativo de alcance descriptivo y relacional de las percepciones obtenidas de los auditores financieros peruanos y su relación con los factores señalados. Los resultados señalan que las mayores valoraciones corresponden a las percepciones de reconocimiento de retos profesionales, necesidad de conocimientos y orientaciones técnicas, uso de especialistas para la aplicación de las normativas sobre el VR. Por otro lado, los factores de nivel académico, experiencia de auditoría y género de los auditores tienen incidencia en las percepciones relacionadas con la necesidad de formación y orientaciones técnicas en normativa del VR, retos y conocimientos necesarios para su auditoría.
\end{abstract}

Palabras clave: auditoría financiera, NIIF, NIA, valor razonable.

Recepción: 27/10/2020. Aprobación: 12/03/2021.

\begin{abstract}
The incorporation of the Fair Value (FV) measurement model into IFRS has led to significant efforts and challenges for financial auditors in the process of auditing the FV estimates in the preparation of financial statements. The objective of the investigation is
\end{abstract}

1 Kiara Chau Miyakawa, Universidad de Piura, Facultad de Ciencias Económicas y Empresariales, Piura, Perú, kiara. chau@udep.edu.pe

2 Julio Hernández Pajares, Universidad de Piura, Facultad de Ciencias Económicas y Empresariales, Lima, Perú, julio. hernandez@udep.edu.pe 
to understand the financial auditor's perception of the FV audit process by considering the most relevant aspects of IFRS 13 and ISA 540. Other objectives are to perceive the views on the challenges posed by these rules and the contribution to the faithful image of financial report, understanding of the strategies that improve the FV audit process and analysing the relationship of these perceptions with the factors of academic level, experience, gender and specialized sector of auditors. In order to achieve these objectives, an exploratory and quantitative study was carried out with a descriptive and relational scope of the perceptions obtained from the Peruvian financial auditors and their relationship with the factors indicated. The results indicate that the highest evaluations correspond to perceptions of recognition of professional challenges, need for knowledge and technical guidance, use of specialists for the application of the regulations on FV. On the other hand, the factors of academic level, audit experience and gender of auditors have an impact on perceptions related to the need for training and technical guidance in FV regulations and the challenges and knowledge required for their audit.

Keywords: financial audit, IFRS, ISA, fair value.

\section{INTRODUCCIÓN}

La adopción de las NIIF ha permitido un proceso de armonización contable internacional, que ha significado un gran desafío para los responsables de la información financiera en el cumplimiento de los requerimientos exigidos por esta normativa internacional (Llopis et al., 2014). Así mismo, este proceso de adopción exige a los auditores el entendimiento de las NIIF considerando una perspectiva más global asociándolas a las normas de auditoría como las NIA, en la formulación de la opinión de auditoría (Ahn et al., 2020; Griffith, 2020; Sosa, 2016).

El concepto de valor razonable (VR) fue incorporado en las NIIF, como criterio para la medición y revelación de determinados elementos, para mejorar la comparabilidad de la información financiera, de acuerdo con la NIIF 13. El Consejo de Normas Internacionales de Contabilidad (IASB) emitió el 2013 esta norma estandarizando los requerimientos de medición y revelación del VR, y es el resultado de esfuerzos conjuntos entre el IASB y el Consejo de Normas de Contabilidad Financiera (FASB) de conciliación entre las normativas. La aplicación de este modelo ha afectado los criterios de medición en los estados financieros, influyendo en las decisiones de los inversores y otros usuarios (Singh, 2015).

Por lo tanto, la estimación del VR por parte de los responsables de la información financiera puede afectar las percepciones sobre el desempeño de una entidad, es así que estudios como los de Bratten et al. (2020), Cannon y Bedard (2017) y Siregar et al. (2020) orientan sus investigaciones al rol del auditor en la auditoría del VR, la capacidad de los auditores para proporcionar el nivel de seguridad requerida de estimaciones con incertidumbre, y conocimiento de las percepciones del VR.

El objetivo general de esta investigación es conocer la percepción de los auditores financieros respecto a la opinión sobre el proceso de auditoría del VR considerando los aspectos más relevantes de las normas NIIF 13 y NIA 540. Otros objetivos son percibir la opinión sobre los retos y 
estrategias de mejora que implican estas normas con el fin de contribuir a la mejora de la imagen fiel de la información financiera y analizar la relación de estas percepciones con los factores de nivel académico, experiencia, género, y sector especializado de los auditores.

Esta investigación presenta además de esta introducción, el marco teórico, el diseño metodológico, análisis de resultados y finalmente las conclusiones.

\section{Antecedentes teóricos}

Como consecuencia de la crisis financiera del 2008, los entes emisores de normas y reguladores presentaron una especial atención en aumentar la responsabilidad del auditor y calidad de trabajo de auditoría con la emisión específica de normativa (Alharasis et al., 2020; Hermanson et al., 2017; Sosa, 2016).

Ante este nuevo entorno regulador el IASB y la Federación Internacional de Contadores (IFAC), plantearon modificaciones en la aplicación de las normas para contadores y auditores con el fin de mejorar la fiabilidad de la información financiera respecto a la aplicación del VR (Devi et al., 2012; Emett et al., 2020; Sosa, 2016). Se revisaron la NIA 200 y NIA 540 sobre objetivos del auditor independiente y auditoría de estimaciones contables, cuyos aspectos más relevantes son la definición de las responsabilidades del auditor en relación a las estimaciones contables, como el entendimiento por parte de los auditores acerca de los modelos de estimación de VR; razonabilidad de los supuestos de medición y, por último, la información de revelación respecto a estas estimaciones.

Por otro lado, en las prácticas contables, se realizó un trabajo conjunto entre el IASB y FASB de unificar conceptos y aspectos importantes sobre el VR creando un marco normativo para asegurar que la definición, requerimientos de medición y revelación comunes, emitiendo la NIIF 13 y la FAS 157 respectivamente (Oyewo, 2020; Sosa, 2016).

\section{Comprensión del Auditor del Concepto del Valor Razonable y su Proceso de Auditoría}

La comprensión del auditor sobre la norma NIIF 13 en las técnicas aplicadas y las estimaciones realizadas por la gerencia son indispensables en el proceso de auditar el VR. Las firmas auditoras influyen en las políticas de medición para las empresas auditadas ya que en el proceso los auditores determinan los riesgos de error material y la razonabilidad de la determinación del criterio de medición (Kumarasiri y Fisher, 2011; Okafor y Osikhena, 2012; Singh, 2015; Sosa, 2016).

Al respecto, el proceso de auditoría de los criterios de medición y revelación del VR mejoran considerablemente la fiabilidad de la información financiera y reducen su asimetría. También, la experiencia, reputación y opinión de auditoría influye en dicha fiabilidad (Cannon y Bedard, 2017; Oliveira et al., 2017; Sosa, 2016).

\section{Desafíos de la Auditoría del Valor Razonable}

Los cambios que ha generado la auditoría del VR encuentran un enfoque más crítico y evaluativo de sus procedimientos y técnicas con la finalidad de alcanzar una fiabilidad a través de la comprobación de los métodos y técnicas utilizados por la entidad y su correcta aplicación (Singh, 
2015; Sosa, 2016). En vista de ello, es un gran reto para los auditores examinar las estimaciones del VR y el posible riesgo que pueda existir errores significativos debido a la posibilidad de sesgo por parte de la dirección en la elección de las técnicas y métodos para la determinación del VR (Bratten et al., 2020; Cannon y Bedard, 2017; Oyewo, 2020).

En la auditoría del VR, los auditores encuentran dificultades en la evaluación de la razonabilidad de las estimaciones de la gerencia del VR, de cotizaciones, precisión de los cálculos, y fijación de materialidad (Kumarasiri y Fisher, 2011; Mendes et al., 2018; Sosa, 2016). Otros problemas en el proceso de auditoría del VR son la falta de conocimientos técnicos, dificultad en la aplicación en diferentes sectores, y una alta incertidumbre en las estimaciones que contribuye a altos riesgos inherentes. (Cannon y Bedard, 2017; Joe et al., 2017; Kohlbeck et al., 2017; Oyewo, 2020).

Estudios sugieren que la aplicación del VR debería limitarse para determinados tipos de activos o pasivos en los que existe un mercado activo y evitar esfuerzos a la utilización de información elaborada internamente con mayor grado de subjetividad, que genera mayores esfuerzos en su auditoria (Kumarasiri y Fisher, 2011; Maisuradze y Vardiashvili, 2016; Mendes et al., 2018).

\section{Propuestas y Estrategias para Mejorar la Auditoría del Valor Razonable por los Auditores}

Ante estos desafíos de la auditoría del VR, se proponen medidas que permitan ayudar en este proceso cuando la metodología aplicada por los directivos se basa en datos no observables e industrias específicas. Por tal motivo, investigaciones como las realizadas por Griffith (2020), Okafor y Osikhena (2012) y Sosa (2016), sugieren que el auditor actualmente debe obtener conocimientos y capacitaciones no solo en contabilidad y auditoría, sino también, en áreas como estadística y economía que permitan desarrollar habilidades y conocimientos para la auditoría a pesar de contar expertos.

Por otro lado, Abdullatif (2016), Cannon y Bedard (2017) y Singh (2015) en sus estudios concluyen que los lineamientos establecidos por las NIA y NIIF son insuficientes en el procedimiento de auditoría del VR. También, Devi et al. (2012) y Oliveira et al. (2017) sugieren un mayor grado de compromiso tanto de los auditores y contadores en las prácticas contables y de auditoría para mejorar la divulgación del VR.

La revisión anterior permite plantear las siguientes preguntas de investigación:

1. ¿Cómo perciben los auditores financieros el efecto del VR en la relevancia de la información financiera según los lineamientos de la NIIF 13 y NIA 540?

2. ¿Qué desafíos perciben los auditores financieros respecto a la auditoría de la información financiera sobre VR?

3. ¿Qué recomendaciones o propuestas proponen los auditores financieros, con la finalidad de superar los desafíos en la auditoría del VR?

Factores sobre la Percepción y Aplicación del Valor Razonable de los Auditores

Estudios realizados por Bratten et al. (2020), Emett et al. (2020), Griffith (2020) y Siregar et al. 
(2020) identificaron posibles factores respecto a atributos personales y profesionales de los auditores financieros que afectan la calidad del proceso de auditoría del valor razonable. Estos corresponden al conocimiento, experiencia y nivel profesional del auditor tienen incidencia en el proceso de auditoría del VR, en relación a los retos y desafíos. Es así que, los auditores más experimentados y con mejores categorías profesionales de auditoría son los más interesados a realizar una constante actualización y entrenamiento para afrontar la auditoría del valor razonable ya que se encuentra directamente ligado a las responsabilidades de su jerarquía (Hermanson et al., 2017; Siregar et al., 2020; Van Linden y Hardies, 2018).

Por lo señalado anteriormente se plantean las siguientes hipótesis:

H1: El nivel académico de los auditores tiene incidencia sobre las valoraciones de auditoria del VR. H2: La experiencia de los auditores tiene incidencia sobre las valoraciones de auditoria del VR.

Por otro lado, Johnson y Powell (1994) estudiaron la variable género, identificando diferencias en la personalidad y comportamiento de hombres y mujeres en relación a la toma de decisiones y aversión al riesgo. En ese sentido, estudios como los realizados por Abdelfattah et al. (2020), Kornberger et al. (2010) y Menezes y Antunes (2015) señalan un cambio en la precepción sobre el dominio masculino en la dirección de las firmas de auditoría, de acuerdo a ello, los estudios señalan una relación de la variable género y la calidad de la auditoría financiera. Se encuentran que las mujeres tienen una mayor aversión al riesgo en el trabajo de auditoría y que las empresas auditadas por mujeres presentan prácticas contables más conservadores (Breesch y Branson, 2009; Chin y Chi, 2008; Menezes y Antunes, 2015).

Por lo señalado anteriormente se plantea la siguiente hipótesis:

H3: El género de los auditores tiene incidencia sobre las valoraciones de auditoría del VR.

Otros estudios encuentran que los auditores de industrias específicas presentan procesos específicos en la auditoría del VR, incidiendo en su calidad y por lo tanto en la fiabilidad de la información financiera auditada. Es así que determinados activos y pasivos de determinadas industrias suelen generar mayores dificultades para los auditores en el proceso de auditoría del VR, como es el caso de activos no financieros en el sector inmobiliario; a diferencia de los activos financieros con mayores datos de entrada observables, con considerable facilidad de estimación (Bratten et al., 2020; Boritz et al., 2020; Hermanson et al., 2017; Mendes et al., 2018).

Por lo señalado anteriormente se propone la siguiente hipótesis:

H4: El sector especializado de los auditores tiene incidencia sobre las valoraciones de auditoria del VR. 


\section{METODOLOGÍA, MATERIALES Y MÉTODOS}

\section{Diseño}

Este estudio exploratorio es de naturaleza cuantitativa sobre el análisis de percepciones obtenidas de encuestas a auditores financieros de firmas de auditoría e independientes. El estudio tiene un alcance descriptivo de la naturaleza de la percepción de los auditores y relacional de los factores de nivel académico, experiencia de auditoría, género y sector especializado de los auditores con las mediciones de las percepciones.

La encuesta se basó en un cuestionario de preguntas cerradas basada en la investigación realizada por Kumarasiri y Fisher (2011), dicho cuestionario basado en 21 cuestiones (Ver Tabla 2) utilizó una escala de Likert de valoración del 1 al 5 que va desde un desacuerdo total, en desacuerdo, ni acuerdo ni desacuerdo, de acuerdo, hasta un acuerdo total, con cada pregunta descritas basada en la teoría revisada. Para medir la consistencia interna de la escala se calculó el coeficiente del alfa de Cronbach con un valor aceptable de 0,766. El cuestionario de la encuesta se diseñó con la herramienta digital de Google Forms, cuyo enlace fue enviado con una invitación por correo electrónico a socios y gerentes de firmas peruanas denominadas big four (Deloitte, EY, KPMG, PWC) para que inviten a otros auditores financieros miembros del staff de auditoría. Asimismo, se invitaron a responder la encuesta a otros auditores independientes peruanos también por correo electrónico. Las invitaciones y respuestas se enviaron y obtuvieron entre los meses de mayo y julio de 2020.

\section{Muestra}

Dado la naturaleza exploratoria del estudio se realizó un muestreo no probabilístico basado en un juicio de invitar a los socios, gerentes, senior y asistentes de las firmas denominadas bif four y a auditores de firmas e independientes. De las invitaciones realizadas por correo electrónico y respuestas obtenidas se obtuvieron 68 respuestas clasificadas según la Tabla 1.

\section{Tabla 1}

Muestra de auditores encuestados según firma.

\begin{tabular}{lccccccc}
\hline \multirow{2}{*}{$\begin{array}{l}\text { Puesto de } \\
\text { Trabajo }\end{array}$} & \multicolumn{9}{c}{ Firma de Auditoria } & \multirow{2}{*}{ Total } \\
\cline { 2 - 7 } Independiente & Deloitte & EY & KPMG & PWC & Otras e Independientes & & \\
\cline { 1 - 7 } Asistente & 0 & 0 & 0 & 0 & 2 & 2 & $3 \%$ \\
Senior & 0 & 1 & 1 & 3 & 0 & 5 & $7 \%$ \\
Gerente & 7 & 11 & 0 & 3 & 0 & 21 & $31 \%$ \\
Socio & 3 & 5 & 1 & 13 & 1 & 23 & $34 \%$ \\
\hline Total & 1 & 8 & 1 & 5 & 2 & 17 & $25 \%$ \\
\hline
\end{tabular}

Nota: Elaboración propia basada en respuestas de encuestas enviadas. 


\section{Variables}

Los componentes o factores respecto a las precepciones sobre el VR comprendidas en las 21 preguntas de la Tabla 2, basadas en la revisión literaria, se obtuvieron de un análisis factorial. Se redujeron las valoraciones a componentes representativos de las percepciones para el posterior estudio relacional mediante pruebas de ANOVA y para ver las incidencias de las variables independientes en los componentes de valoraciones.

Las variables independientes que se van a considerar para el análisis relacional, obtenidas de los datos de las encuestas, son la variable categórica de género de los auditores encuestados clasificados en las categorías de masculino y femenino; la variable ordinal de nivel académico que considera los grados académicos de bachiller, licenciado y master; la variable categórica de años de experiencia que considera seis intervalos que van desde una experiencia menor a 5 años hasta la máxima de mayor de 25 años; y finalmente la variable de categoría de sector especializado de experiencia del auditor que comprenden agroindustria, construcción e inmobiliario, servicios públicos, petróleo y minerales, financiero, industria.

\section{RESULTADOS}

\section{Análisis Descriptivo}

La Tabla 2 muestra los resultados obtenidos de las encuestas sobre las precepciones de los auditores agrupados en tres categorías teóricas. La primera agrupación sobre las percepciones de los auditores en relación al efecto del VR en la información financiera y sus lineamientos, que muestran en términos generales que los auditores opinan mayormente estar entre "ni acuerdo ni descuerdo" y "de acuerdo", en la utilidad del VR en la fiabilidad de la información financiera y los lineamientos de la normativa, pero sobre todo en conocer los procesos de medición y auditoria del VR (ver preguntas 1, 2 y 8 con mayor valoración). Las opiniones en "de acuerdo" valoran la información como más relevante y útil para la toma de decisiones, a pesar de las estimaciones del VR. (Kumarasiri y Fisher, 2011; Singh, 2015; Siregar et al., 2020).

La segunda categoría de preguntas se refiere a los retos y desafíos que perciben los auditores financieros respecto a la auditoría de VR. Las valoraciones que van entre "ni acuerdo ni descuerdo" y "de acuerdo". Las de conformidad concuerdan estudios de Oyewo (2020) y Singh (2015) quienes afirman que auditar bajo el VR demanda mayores esfuerzos y retos como el tiempo y conocimientos técnicos para la verificabilidad de sus estimaciones, como señalan las altas valoraciones de las preguntas 10, 11 y 12. También se valora las dificultades en medir el VR bajo datos no observables que requieren la incorporación frecuente de estimaciones y condiciones futuras como indican las valoraciones de las preguntas 14, 15 y 16. Finalmente la última categoría es sobre las propuestas de estrategias para mejorar la auditoría del VR, las valoraciones reconocen la necesidad de una educación continua y especializada sobre el VR, el apoyo de especialistas expertos y mejores orientaciones técnicas de la normativa como muestran las valoraciones de las preguntas 18 y 19 . 


\section{Tabla 2}

\section{Estadísticos descriptivos de las preguntas valorados en las encuestas.}

\begin{tabular}{|c|c|c|}
\hline Preguntas & Media & $\begin{array}{l}\text { Desviación } \\
\text { estándar }\end{array}$ \\
\hline \multicolumn{3}{|l|}{ Percepciones sobre la relación entre VR e información financiera } \\
\hline $\begin{array}{l}\text { 1. El VR es una mejor base para la presentación de información financiera que el } \\
\text { Costo Histórico }(\mathrm{CH})\end{array}$ & 4.06 & 0.991 \\
\hline $\begin{array}{l}\text { 2. Como base para la medición en los Estados Financieros, el VR permite una } \\
\text { mejor relevancia de la información financiera que el realizado por el CH }\end{array}$ & 4.09 & 0.768 \\
\hline $\begin{array}{l}\text { 3. Los Estados Financieros basado en VR son más comprensibles para los usuarios } \\
\text { que los preparados bajo el CH }\end{array}$ & 3.29 & 0.963 \\
\hline $\begin{array}{l}\text { 4. Existe una mayor relevancia de la información financiera basados en el VR a } \\
\text { pesar de la reducción de la fiabilidad de dicha información }\end{array}$ & 3.51 & 1.015 \\
\hline $\begin{array}{l}\text { 5. En general, los Estados Financieros preparados bajo el VR son más útiles para } \\
\text { los usuarios que los preparados bajo el CH }\end{array}$ & 3.71 & 0.899 \\
\hline $\begin{array}{l}\text { 6. Los auditores de acuerdo a su formación y experiencia tienen un claro } \\
\text { entendimiento de los requerimientos de la NIIF } 13\end{array}$ & 3.71 & 1.185 \\
\hline $\begin{array}{l}\text { 7. Los auditores de acuerdo a su formación y experiencia tienen un claro } \\
\text { entendimiento de los requerimientos de la NIA } 540\end{array}$ & 3.68 & 1.177 \\
\hline $\begin{array}{l}\text { 8. Antes de auditar los elementos de los estados financieros medidos a VR, los } \\
\text { auditores necesitan conocer los procesos de medición utilizados por el cliente }\end{array}$ & 4.57 & 0.581 \\
\hline \multicolumn{3}{|l|}{ Percepciones de Retos y desafíos de la auditoría del VR } \\
\hline $\begin{array}{l}\text { 9. La auditoría de los informes financieros basados en el VR implica un mayor } \\
\text { reto profesional para los auditores }\end{array}$ & 4.41 & 0.629 \\
\hline $\begin{array}{l}\text { 10. El mayor reto al que se enfrentan los auditores en relación al VR es la } \\
\text { verificabilidad de sus estimaciones }\end{array}$ & 4.40 & 0.626 \\
\hline $\begin{array}{l}\text { 11. Los auditores necesitan de conocimientos técnicos más especializados para } \\
\text { auditar el VR }\end{array}$ & 4.22 & 0.730 \\
\hline $\begin{array}{l}\text { 12. La determinación de las estimaciones a VR es una tarea que requiere tiempo } \\
\text { para su verificación }\end{array}$ & 3.60 & 1.024 \\
\hline $\begin{array}{l}\text { 13. Las técnicas utilizadas para determinar el VR difieren en relación a la actividad } \\
\text { económica de la empresa auditada }\end{array}$ & 3.37 & 1.050 \\
\hline $\begin{array}{l}\text { 14. La mayoría de activos y pasivos significativos no se negocian en mercados } \\
\text { activos, sino que se estiman en base a datos no observables }\end{array}$ & 3.18 & 1.119 \\
\hline $\begin{array}{l}\text { 15. Los activos y pasivos se valoran utilizando hipótesis de gestión y modelos } \\
\text { matemáticos }\end{array}$ & 3.66 & 0.745 \\
\hline $\begin{array}{l}\text { 16. Las oportunidades disponibles para que los auditores obtengan conocimientos } \\
\text { sobre las mediciones del VR son limitadas }\end{array}$ & 3.00 & 0.962 \\
\hline $\begin{array}{l}\text { 17. Las mediciones del VR incorporan frecuentemente estimaciones de eventos y } \\
\text { condiciones futuras }\end{array}$ & 3.93 & 0.834 \\
\hline \multicolumn{3}{|l|}{$\begin{array}{l}\text { Percepciones sobre Propuestas y Estrategias para afrontar retos de auditoría } \\
\text { de VR }\end{array}$} \\
\hline $\begin{array}{l}\text { 18. Los auditores deberían recibir una formación profesional más completa sobre } \\
\text { el VR }\end{array}$ & 4.32 & 0.531 \\
\hline $\begin{array}{l}\text { 19. Es necesario el apoyo de especialistas expertos en el proceso de auditoría del } \\
\text { VR }\end{array}$ & 4.12 & 0.764 \\
\hline $\begin{array}{l}\text { 20. El VR debería limitarse a los activos y pasivos con disponibilidad en mercados } \\
\text { activos }\end{array}$ & 2.63 & 1.171 \\
\hline $\begin{array}{l}\text { 21. Es necesario el desarrollo de mejores orientaciones técnicas de normativa } \\
\text { contable y auditoria del VR }\end{array}$ & 4.04 & 0.781 \\
\hline
\end{tabular}

Nota: Elaboración propia. 


\section{Análisis relacional}

La prueba de análisis factorial exploratorio permitió reducir las variables de valoración a cinco componentes. Con una proporción de varianza explicada del $60 \%$, se obtuvo una confiabilidad con un estadístico de 0,595 de adecuación muestral y una significancia de 0 (sig.<0.05) de rechazo de hipótesis nula de independencia de variables, según pruebas de Kaiser-Meyyer-Olkin (KMO) y Bartlett respectivamente. Los componentes sobre las valoraciones, obtenidos de análisis factorial se observan en las Figuras 1, 2, 3 y 4 que muestran las medias de los componentes para las cuatro variables categóricas independientes.-

El primer componente "VR e Información Financiera" representa las valoraciones que dan los auditores a la fiabilidad, utilidad y relevancia de información financiera preparada bajo modelos de VR. El segundo componente "Retos y Conocimientos" agrupa las valoraciones sobre los retos profesionales, conocimientos técnicos y capacidad de verificabilidad de las estimaciones de VR. El tercer componente "Formación y Experiencia en Normativas" representa las valoraciones referidas a la necesidad de que los auditores requieren formación y experiencia para la auditoria del VR. El cuarto componente de “Orientaciones Técnicas y Estimaciones" corresponde a las valoraciones sobre la necesidad y respuestas de manejar las orientaciones técnicas, conocimiento de procesos de estimaciones de VR. Finalmente, el último componente "Limitaciones de técnicas y conocimientos" está referido a las valoraciones sobre las limitaciones en información y conocimientos de medición de VR considerando los sectores empresariales específicos. Para el análisis de incidencia se aplicaron pruebas de ANOVA, cuyos resultados se observan en la Tabla 3 que se analizaron con los resultados de las Figuras 1 a 4 sobre las percepciones por componentes factoriales.

\section{Figura 1}

Percepciones según nivel académico.

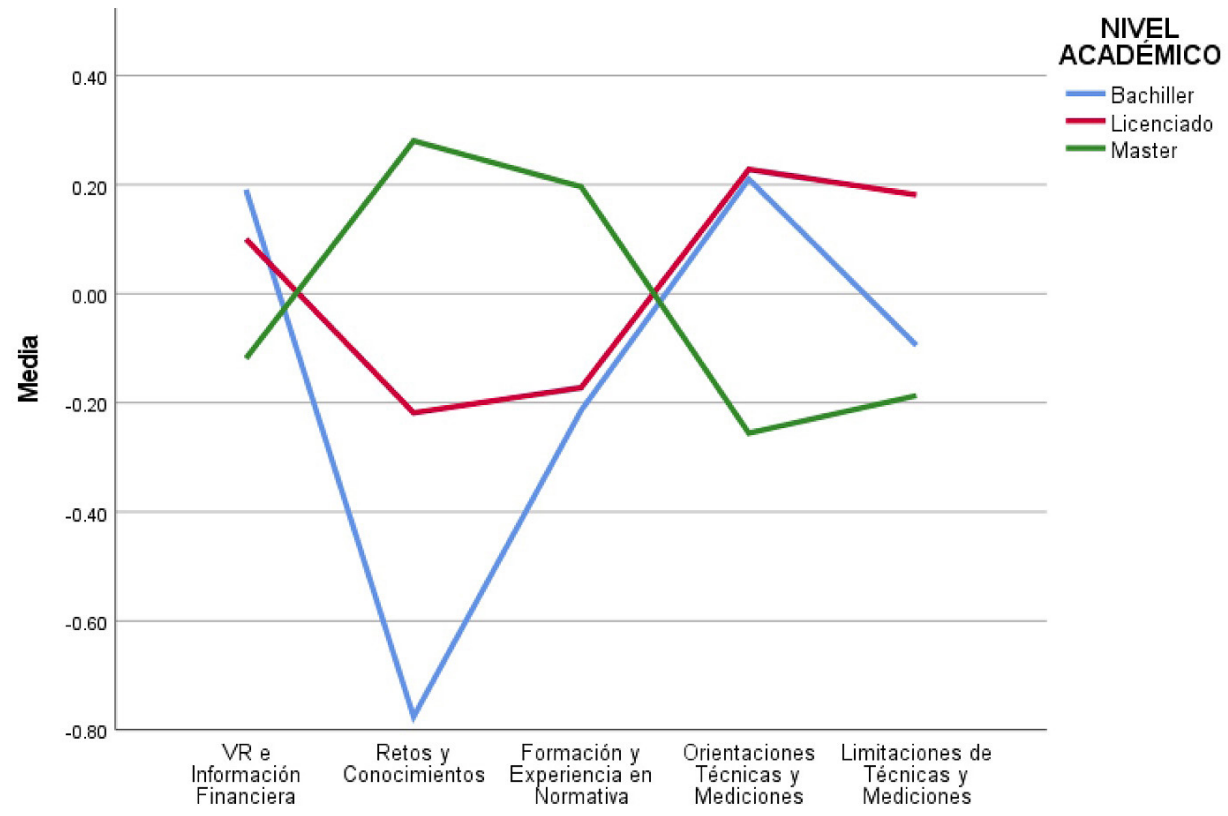

Nota: Elaboración propia basada en resultados de encuestas. 


\section{Figura 2}

Percepciones según años de experiencia en auditoría.

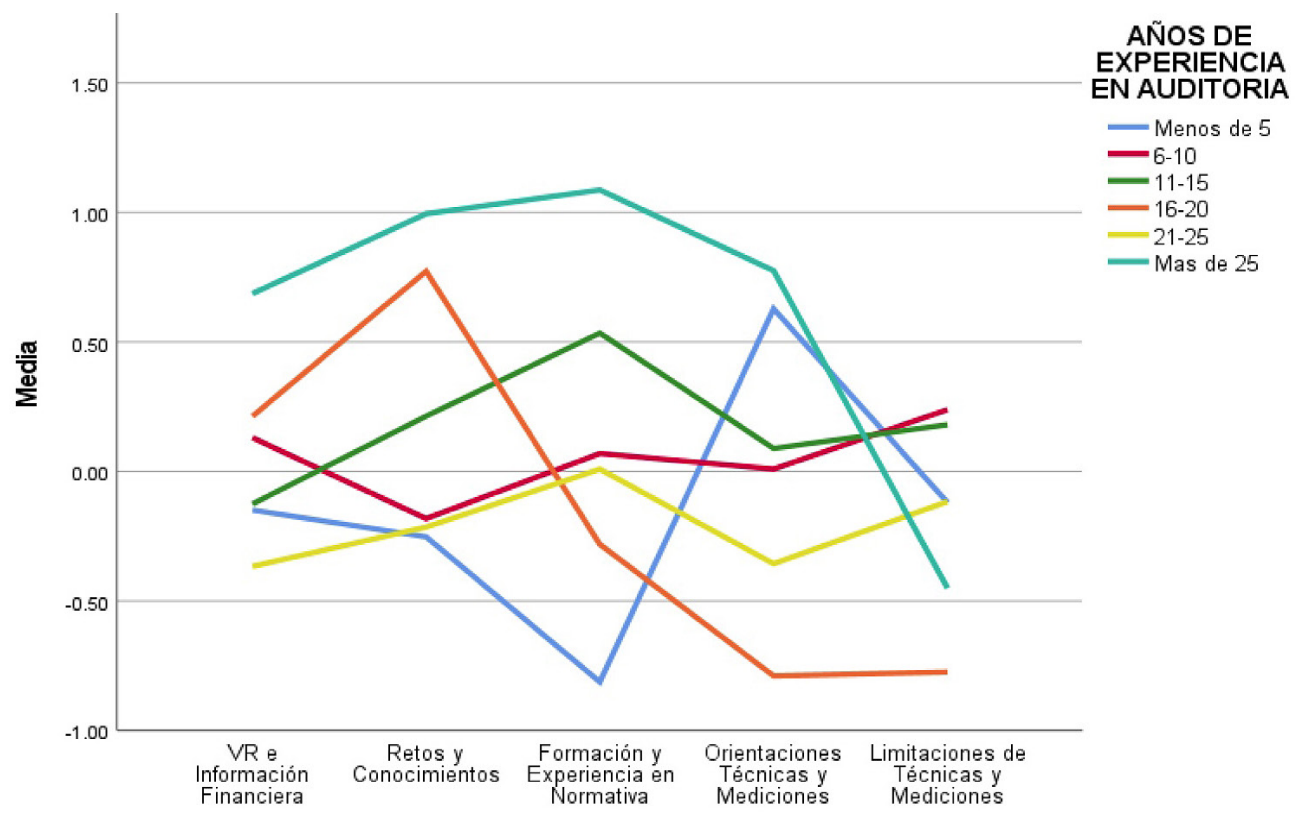

Nota: Elaboración propia basada en resultados de encuestas.

\section{Figura 3}

\section{Percepciones según género.}

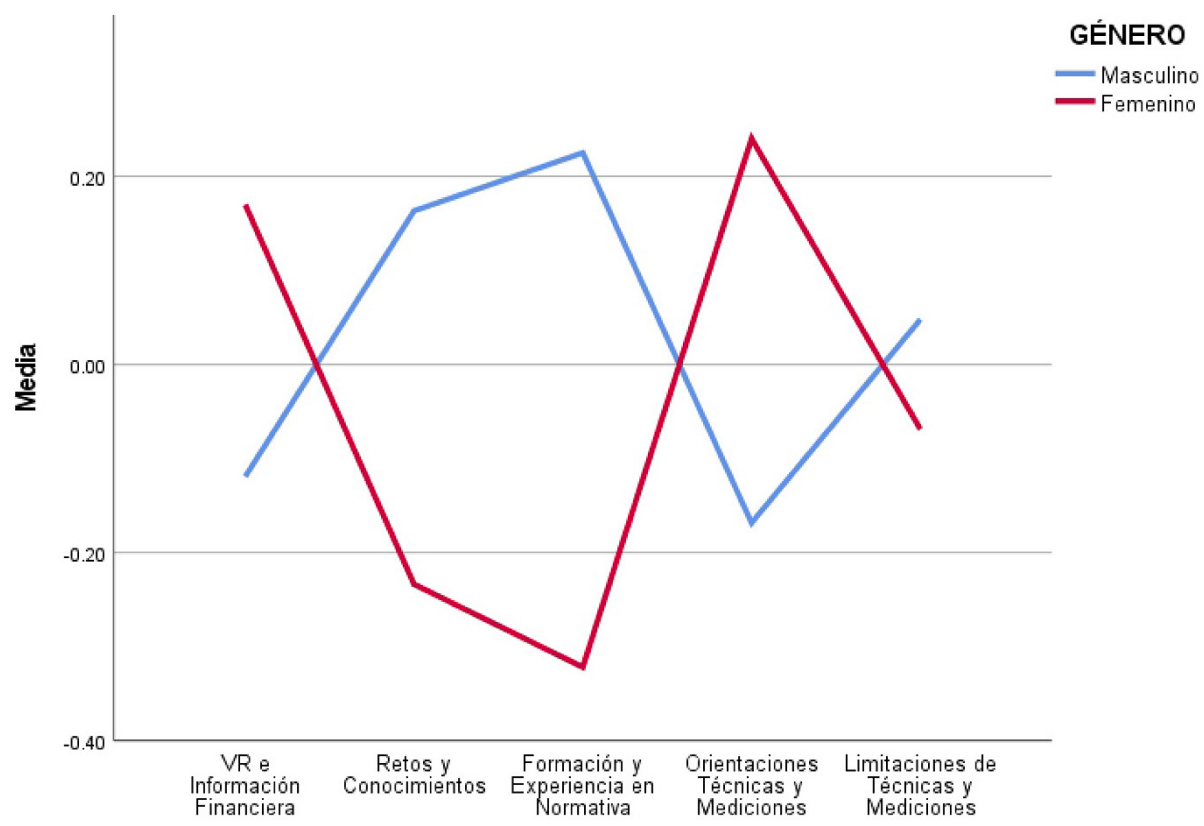

Nota: Elaboración propia basada en resultados de encuestas. 


\section{Figura 4}

Percepciones según sector empresarial.

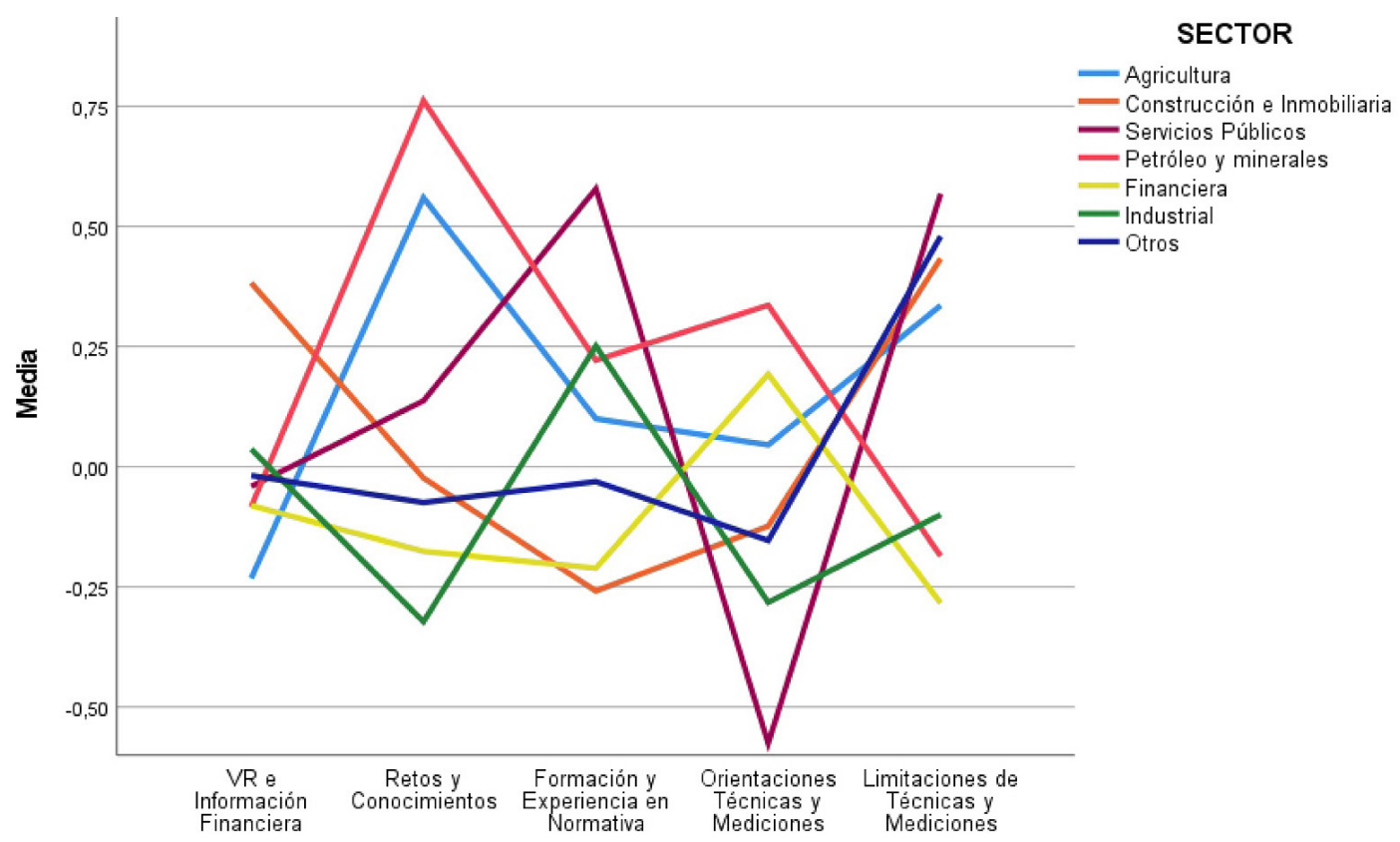

Nota: Elaboración propia basada en resultados de encuestas. 
Tabla 3

Prueba ANOVA de Factores sobre Componentes de Valoración.

\begin{tabular}{|c|c|c|c|c|c|c|}
\hline \multicolumn{2}{|l|}{ Variable Independiente Nivel Académico } & \multirow{2}{*}{$\begin{array}{r}\begin{array}{c}\text { Suma de } \\
\text { cuadrados }\end{array} \\
0,863\end{array}$} & \multirow{2}{*}{$\begin{array}{r}\text { gl. } \\
2\end{array}$} & \multirow{2}{*}{$\begin{array}{r}\text { Media } \\
0,431\end{array}$} & \multirow{2}{*}{$\frac{F}{0,424}$} & \multirow{2}{*}{$\begin{array}{l}\text { Sig. } \\
0,656\end{array}$} \\
\hline & Entre grupos & & & & & \\
\hline VR e Información Financiera & En grupos & 66,137 & 65 & 1,017 & & \\
\hline \multirow{2}{*}{ Retos y Conocimientos } & Entre grupos & 5,340 & 2 & 2,670 & 2,815 & $0,067^{*}$ \\
\hline & En grupos & 61,660 & 65 & 0,949 & & \\
\hline \multirow{2}{*}{ Formación y Experiencias en Normativas } & Entre grupos & 2,332 & 2 & 1,166 & 1,172 & 0,316 \\
\hline & En grupos & 64,668 & 65 & 0,995 & & \\
\hline \multirow{2}{*}{ Orientaciones Técnicas y Mediciones } & Entre grupos & 3,950 & 2 & 1,975 & 2,036 & 0,139 \\
\hline & En grupos & 63,050 & 65 & 0,970 & & \\
\hline \multirow{2}{*}{ Limitaciones de Técnicas y Mediciones } & Entre grupos & 2,255 & 2 & 1,128 & 1,132 & 0,329 \\
\hline & En grupos & 64,745 & 65 & 0,996 & & \\
\hline \multicolumn{7}{|c|}{ Variable Independiente Años de Experiencia } \\
\hline \multirow{2}{*}{ VR e Información Financiera } & Entre grupos & 3,476 & 5 & 0,695 & 0,679 & 0,641 \\
\hline & En grupos & 63,524 & 62 & 1,025 & & \\
\hline \multirow{2}{*}{ Retos y Conocimientos } & Entre grupos & 8,688 & 5 & 1,738 & 1,848 & 0,117 \\
\hline & En grupos & 58,312 & 62 & 0,941 & & \\
\hline \multirow{2}{*}{ Formación y Experiencias en Normativas } & Entre grupos & 12,793 & 5 & 2,559 & 2,926 & $0,020^{* *}$ \\
\hline & En grupos & 54,207 & 62 & 0,874 & & \\
\hline \multirow{2}{*}{ Orientaciones Técnicas y Mediciones } & Entre grupos & 10,866 & 5 & 2,173 & 2,400 & $0,047^{* *}$ \\
\hline & En grupos & 56,134 & 62 & 0,905 & & \\
\hline \multirow{2}{*}{ Limitaciones de Técnicas y Mediciones } & Entre grupos & 6,834 & 5 & 1,367 & 1,408 & 0,234 \\
\hline & En grupos & 60,166 & 62 & 0,970 & & \\
\hline \multicolumn{7}{|l|}{ Variable Independiente Género } \\
\hline \multirow{2}{*}{ VR e Información Financiera } & Entre grupos & 1,378 & 1 & 1,378 & 1,386 & 0,243 \\
\hline & En grupos & 65,622 & 66 & 0,994 & & \\
\hline \multirow{2}{*}{ Retos y Conocimientos } & Entre grupos & 2,596 & 1 & 2,596 & 2,660 & 0,108 \\
\hline & En grupos & 64,404 & 66 & 0,976 & & \\
\hline \multirow{2}{*}{ Formación y Experiencias en Normativas } & Entre grupos & 4,925 & 1 & 4,925 & 5,237 & $0,025^{* *}$ \\
\hline & En grupos & 62,075 & 66 & 0,941 & & \\
\hline \multirow{2}{*}{ Orientaciones Técnicas y Mediciones } & Entre grupos & 2,750 & 1 & 2,750 & 2,825 & $0,098^{*}$ \\
\hline & En grupos & 64,250 & 66 & 0,973 & & \\
\hline \multirow{2}{*}{ Limitaciones de Técnicas y Mediciones } & Entre grupos & 0,225 & 1 & 0,225 & 0,222 & 0,639 \\
\hline & En grupos & 66,775 & 66 & 1,012 & & \\
\hline \multicolumn{7}{|l|}{ Variable Independiente Sector } \\
\hline \multirow{2}{*}{ VR e Información Financiera } & Entre grupos & 1,824 & 6 & 0,304 & 0,284 & 0,942 \\
\hline & En grupos & 65,176 & 61 & 1,068 & & \\
\hline & Entre grupos & 8,495 & 6 & 1,416 & 1,476 & 0,201 \\
\hline Retos y Conocimientos & En grupos & 58,505 & 61 & 0,959 & & \\
\hline Formación y Fyno & Entre grupos & 4,006 & 6 & 0,668 & 0,647 & 0,693 \\
\hline Formacion y Experiencias en Normativas & En grupos & 62,994 & 61 & 1,033 & & \\
\hline & Entre grupos & 4,163 & 6 & 0,694 & 0,674 & 0,671 \\
\hline Orientaciones Técnicas y Mediciones & En grupos & 62,837 & 61 & 1,030 & & \\
\hline nitaciones de Té & Entre grupos & 6,716 & 6 & 1,119 & 1,133 & 0,354 \\
\hline Limitaciones de Técnicas y Mediciones & En grupos & 60,284 & 61 & 0,988 & & \\
\hline
\end{tabular}

${ }^{* *} \mathrm{p}<0.05,{ }^{*} \mathrm{p}<0.10$

Nota: Elaboración propia 
La Figura 1, muestra los componentes de las percepciones según el nivel académico. Los auditores con menor formación como bachilleres valoran las orientaciones técnicas y conocimientos para las mediciones del VR en el proceso de auditoría, al igual que los licenciados que además valoran afrontar las limitaciones en información y conocimientos de técnicas de medición de VR considerando los sectores empresariales específicos. Distinto al caso de los auditores másteres con mayor formación que dan importancia a los retos que implican las mediciones del VR y a la experiencia y formación en el manejo de la normativa (Hermanson et al., 2017; Griffith, 2020). La prueba de ANOVA de la Tabla 3 confirma que el factor de nivel académico incide significativamente $(p<0,10)$ en el componente de "Retos y Conocimientos", la media de valoración difiere significativamente para los auditores según nivel académico, por lo que no rechazamos la hipótesis H1 para este componente.

La Figura 2 muestra que los auditores con mayores años de experiencia valoran los retos y desafíos de la auditoría del VR y la necesidad de formación y experiencia en la normativa para una mejor calidad de auditoría (Soleimany et al., 2017; Van Linden y Hardies, 2018), caso contrario a los de menor experiencia que valoran la necesidad de mejores conocimientos de procesos de estimaciones de VR que no son obtenidas de mercados activos, que requieren un mayor esfuerzo de verificación (Bratten et al., 2020; Singh, 2015). La prueba de ANOVA confirma que el factor de los años de experiencia incide significativamente $(p<0,05)$ en los componentes de "Formación y Experiencias en Normativas" y “Orientaciones Técnicas y Mediciones" por lo que no rechazamos la hipótesis $\mathrm{H} 2$ para dichos componentes.

Se observa en la Figura 3 que las percepciones según el género de los auditores son distintas para los componentes de valoración de retos, conocimientos y formación en normativa del VR, donde la valoración es mayor en los hombres. Para el componente de necesidad de orientaciones técnicas que se requieren para afrontar mejor la auditoría del VR es más valorado por las mujeres. La prueba de ANOVA confirma que el factor de género incide significativamente $(p<0,05, p<0,10)$ en los componentes de "Formación y Experiencias en Normativas" y "Orientaciones Técnicas y Mediciones", por lo que no rechazamos la hipótesis H3 para estos componentes. Estos hallazgos se relacionan con que las mujeres auditoras buscan disminuir el riesgo de trabajo de auditoría y se orientan a prácticas contables conservadoras (Abdelfattah et al., 2020; Breesch y Branson, 2009).

Finalmente, el sector empresarial de acuerdo a la Figura 4 muestra que el nivel de valoración es mayor en el componente de "Retos y conocimientos" que afrontan los auditores de las industrias especializadas de agroindustria, y petróleo y minería que requieren estimaciones de VR específicas. Los auditores de la industria de agua, energía y gas (servicios públicos) presenta una alta valoración de la formación y experiencias en la aplicación de normativas de auditoría del VR. Son valorados el reconocimiento de limitaciones para las mediciones de VR en sectores de construcción e inmobiliarios y de servicios. Sin embargo, la prueba de ANOVA no encuentra una incidencia significativa del sector en las valoraciones, por lo que rechazamos la hipótesis H4. Resultados que difieren de estudios que encuentran incidencia de la especialización de industria (Bratten et al., 2020; Maisuradze y Vardiashvili, 2016). 


\section{DISCUSIÓN Y CONCLUSIONES}

Este estudio buscó contribuir a la investigación sobre la percepción de los auditores con respecto a la auditoría del VR, de acuerdo a la normativa, para mejorar las características de relevancia y fiabilidad en la elaboración y auditoría de los estados financieros, lo que ha implicado una mayor responsabilidad de contadores y auditores (Alharasis et al., 2020; Sosa, 2016).

De acuerdo a los resultados obtenidos las percepciones de los auditores señalan, en su mayoría, la importancia de aplicación del modelo del VR en la preparación de la información financiera, la cual favorece su relevancia y utilidad en la toma de decisiones de los usuarios (Kumarasiri y Fisher, 2011; Singh, 2015). Sin embargo, lo anterior no contradice que los auditores reconozcan un reto profesional afrontar la verificación de las estimaciones del VR, más aún cuando no corresponde a un valor de mercado objetivo, y para ello se requiera de formación en conocimientos técnicos acerca de la normativa contable y de auditoría y de apoyo en especialistas expertos (Bratten et al., 2020; Cannon y Bedard, 2017; Griffith, 2020; Soleimany et al., 2017).

El estudio también encuentra que los auditores de menor experiencia, nivel académico, mujeres y de sectores especializados son los que reconocen una necesidad de formación y orientaciones técnicas para la auditoría del VR, que disminuya el riesgo de trabajo de auditoría (Oliveira et al., 2017; Soleimany et al., 2017), contrario a los auditores con mayor experiencia en años y nivel académico que afrontan la auditoría del VR como un reto profesional y que requiere de experiencia en los conocimientos y técnicas de auditoría, es decir, con una menor adversidad al riesgo (Hermanson et al., 2017; Van Linden y Hardies, 2018).

Se considera que esta investigación exploratoria, aporta en el estudio sobre la práctica profesional en auditoría, en un área que requiere de formación y experiencia para una adecuada auditoría del modelo del VR y continúa siendo un desafío para auditores de firmas de auditoría e independientes. Como implicancia del trabajo se requieren futuros estudios con un mayor número de auditores y firmas de distintos países en la región, para obtener resultados que confirmen los antecedentes teóricos.

\section{REFERENCIAS BIBLIOGRÁFICAS}

Abdelfattah, T., Elmahgoub, M., y Elamer, A. A. (2020). Female audit partners and extended audit reporting: UK evidence. Journal of Business Ethics. https: / / doi.org/10.1007 / s10551-02004607-0

Ahn, J., Hoitash, R., y Hoitash, U. (2020). Auditor task-specific expertise: The case of fair value accounting. The Accounting Review, 95(3), 1-32.

Alharasis, E. E., Prokofieva, M., Alqatamin, R. M., y Clark, C. (2020). Fair Value Accounting and Implications for the Auditing Profession: Historical Overview. Accounting and finance Research, 9(3), 31-52.

Abdullatif, M. (2016). Auditing fair value estimates in developing countries: The case of Jordan. Asian Journal of Business and Accounting, 9(2), 101-140.

Boritz, J. E., Kochetova, N. V., Robinson, L. A., y Wong, C. (2020). Auditors' and specialists' views about the use of specialists during an audit. Behavioral Research in Accounting, 32(2), 15-40. 
Bratten, B., Causholli, M., y Myers, L. A. (2020). Fair value exposure, auditor specialization, and banks' discretionary use of the loan loss provision. Journal of Accounting, Auditing $\mathcal{E}$ Finance, 35(2), 318-348.

Breesch, D. y Branson, J. (2009) The Effects of Auditor Gender on Audit Quality, The IUP Journal of Accounting Research \& Audit Practices, 8 (3/4), 78-107.

Cannon, N. H., y Bedard, J. C. (2017). Auditing challenging fair value measurements: Evidence from the field. The Accounting Review, 92(4), 81-114.

Chin, C. y Chi, H. (2008) Sex Matters: Gender Differences in Audit Quality. Working paper presented at the Annual Meeting of the American Accounting Association, August 3-6, Anaheim.

Devi, R., Devi, J., Kumar, R., y Taylor, C. (2012). Accountant and user perceptions of fair value accounting: Evidence from Fiji. Global Journal of Business Research, 6(3), 93-102.

Emett, S. A., Kaplan, S. E., Mauldin, E., y Pickerd, J. S. (2020). Auditing with Data and Analytics: External Reviewers' Judgments of Audit Quality and Effort, SSRN. http:/ / dx.doi. org / 10.2139 / ssrn.3544973

Griffith, E. (2020). Auditors, specialists, and professional jurisdiction in audits of fair values. Contemporary Accounting Research, 37(1), 245-276.

Hermanson, S. D., Kerler III, W. A., y Rojas, J. D. (2017). An Analysis of Auditors' Perceptions Related to Fair Value Estimates. Journal of Corporate Accounting \& Finance, 28(3), 18-37.

Joe, J. R., Vandervelde, S. D., y Wu, Y. J. (2017). Use of high quantification evidence in fair value audits: Do auditors stay in their comfort zone? The Accounting Review, 92(5), 89-116.

Johnson, J. E. y Powell, P. L. (1994). Decision Making, Risk and Gender: Are Managers Different? British Journal of Management, 5 (2), 123-138.

Kohlbeck, M., Smith, T., y Valencia, A. (2017). Auditors and net transfers of Level 3 fair-valued financial instruments. Advances in Accounting, 36, 27-39.

Kumarasiri, J., y Fisher, R. (2011). Auditors' perceptions of fair-value accounting: developing country evidence. International Journal of Auditing, 15(1), 66-87.

Kornberger, M., Carter, C., y Ross-Smith, A. (2010). Changing gender domination in a big four accounting firm: Flexibility, performance and client service in practice. Accounting, Organizations and Society, 35(8), 775-791.

Llopis, R. M., Becerra, O. A., Vásquez, J. C., y Casinelli, H. P. (2014). El proceso de convergencia con las Normas Internacionales de Información Financiera en España, Perú y Argentina. Contabilidad y Negocios, 9(18), 6-26.

Maisuradze, M., y Vardiashvili, M. (2016). Main aspects of measurement of the fair value of nonfinancial assets. Economic and Social Development: Book of Proceedings, 385-391.

Menezes, T., y Antunes, F. (2015). Audit quality: does gender composition of audit firms matter? Spanish Journal of Finance and Accounting, 44(3), 264-297.

Mendes, P. C., Niyama, J. K., y Silva, C. A. (2018). The Perception of Auditors in the Measurement of Instruments Financial Institutions at Fair Value in Financial Institutions. Brazilian Business Review, 15(4), 363-381.

Okafor, C., y Osikhena, K. (2012). Perceptions of fair value accounting: Evidence from Nigeria. Journal of Research in National Development, 10(3), 417-432.

Oyewo, B. (2020). Post-implementation challenges of fair value measurement (IFRS 13): some empirical evidence. African Journal of Economic and Management Studies, 11 (4), 587-607.

Oliveira, N. C., Nakao, S. H., y Nardi, P. C. (2017). Análise da influência das firmas de auditoria na divulgação de informações em notas explicativas. Revista Base, 14 (2), 139-154. 
Singh, J. P. (2015). Fair Value Accounting: A Practitioner's Perspective. IUP Journal of Accounting Research $\mathcal{E}$ Audit Practices, 14 (2), 53-65.

Soleimany G. R., y Nasab, N. (2017). Auditing Challenging Fair Value and Other Estimates. Journal of Accounting Research, 6(4), 128-144.

Siregar, S. V., Djakman, C. D., Mita, A. F., y Ningrum, A. S. (2020). Perceptions of Practitioners, Auditors, and Academics on IFRS Convergence in Indonesia. En Advanced Issues in the Economics of Emerging Markets. Emerald Publishing Limited.

Sosa, E. (2016). La auditoría de valores razonables: riesgos y desafíos para el auditor. Pensamiento actual, 16(27), 297-313.

Van Linden, C., y Hardies, K. (2018). Entrance requirements to the audit profession within the EU and audit quality. International Journal of Auditing, 22 (3), 360-373.

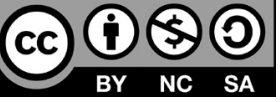

Esta obra está bajo una licencia de

Creative Commons Atribución-NoComercial-CompartirIgual 4.0 Internacional 